\title{
Coordination between Extrinsic Extracellular Matrix Cues and Intrinsic Responses to Orient the Centrosome in Polarizing Cerebellar Granule Neurons
}

\author{
Shailesh Kumar Gupta, ${ }^{1}$ Karina F. Meiri, ${ }^{2}$ Kashif Mahfooz, ${ }^{1}$ Upasna Bharti, ${ }^{1}$ and Shyamala Mani ${ }^{1}$ \\ ${ }^{1}$ National Brain Research Center, Manesar, Haryana 122 050, India, and ${ }^{2}$ Department of Anatomy and Cellular Biology, Tufts University School of Medicine, \\ Boston, Massachusetts 02111
}

\begin{abstract}
Successful axon targeting during development is critically dependent on directionality of axon extension and requires coordination between the extrinsic cues that provide spatial information to the axon and the intrinsic responses that regulate structural specification of the axon during neuronal polarization. How these responses are coordinated is unclear but are known to involve aligning the centrosome with the base of the emerging axon. We have used a novel in vitro micropatterning assay that spatially segregates the extrinsic cues used by polarizing cerebellar granule cells to orient axon extension and used it to investigate the signaling mechanisms responsible for coordinating centrosome positioning with intrinsic responses. The results show that, when laminin and/or vitronectin are used as spatially restricted cues in association with substrate-associated sonic hedgehog, they are sufficient to induce cell cycle arrest, that laminin and vitronectin then induce integrin-mediated signaling that upregulates phosphoinositide- 3 kinase and PKC function to produce phosphatidylinositol 3,4,5-trisphosphate (PIP3) that is associated with the centrosome, that this PIP3 can interact with PKCphosphorylated growth-associated protein GAP-43, and that PKC-phosphorylated GAP-43 in turn is required for positioning Par6, Cdc42, and IQGAP1, all intrinsic response components, in proximity to the centrosome, such that, in the absence of GAP-43, they are mislocalized and microtubules are not oriented appropriately. We conclude from these results that GAP-43 plays an important role in coordinating extrinsic signaling and intrinsic responses in polarizing cerebellar granule neurons.
\end{abstract}

\section{Introduction}

Development of cerebellar granule neurons has been well characterized, and their in vitro accessibility makes them an ideal system in which to study regulation of neuronal polarization (Powell et al., 1997). Previous studies have shown the important role played by the centrosome: when granule cells exit the cell cycle, the centrosome is positioned at the pole from which the axon will emerge together with signaling molecules important in establishing the structural polarity of the axon such as cell division cycle 42 (Cdc42) and the partitioning defective (Par) complex (Zmuda and Rivas, 1998; Higginbotham et al., 2006). This positioning and subsequent emergence of the leading process dictates the direction of axon extension, which is key for correct target identification (Solecki et al., 2004). Previous studies using hippocampal cells in culture have shown an association between the position of the centrosome as polarization is initiated and the orientation of the spindle during the last mitotic division, leading to the hypothesis that neuronal polarity is driven intrinsically via

Received Aug. 26, 2009; revised Dec. 17, 2009; accepted Jan. 6, 2010

S.K.G. and K.M. were supported by the Council of Scientific and Industrial Research, Government of India fellowship awards. Funding for the study was provided by the National Brain Research Centre (S.M.) and National Institutes of Health Grant NS33118 (K.F.M.). We thank Dr. Pierre Gressens for critical reading of this manuscript. We also thank Dr. David Juncker for kindly providing us with the lithographically fabricated silicon masters.

Correspondence should be addressed to Dr. Shyamala Mani at her present address: Centre for Neuroscience, Indian Institute of Science, Bangalore, 560012 India. E-mail: shyamala@cns.iisc.ernet.in.

DOI:10.1523/JNEUROSCI.4218-09.2010

Copyright $\odot 2010$ the authors $\quad 0270-6474 / 10 / 302755-12 \$ 15.00 / 0$ microtubule dynamics (de Anda et al., 2005; Calderon de Anda et al., 2008). Although this may be true in a simple in vitro situation in which there are no extrinsic cues to dictate axon extension, it cannot be true in vivo in which the direction of axon extension is critical for correct target identification. However, the underlying mechanisms that coordinate responsivity to spatially localized extrinsic cues that dictate where the axon will be extended with the intrinsic molecules that regulate the cytoskeletal response are not well understood. Evidence suggests that actin regulation is also involved: key components of the polarity signaling complex that are responsive to extrinsic cues, i.e., Cdc42 and Par3/Par6, are linked to actin rather than tubulin polymerization, and, when they indirectly respond to spatially localized extrinsic cues, such as during directional outgrowth of the growth cone, they cause localized actin assembly that is then followed by microtubule stabilization (Luo et al., 1997; Zmuda and Rivas, 2000; Alberts et al., 2006; Chen et al., 2006b). The results suggest that other as yet unidentified proteins that are directly responsive to spatially localized extrinsic cues are required to coordinate actin and tubulin regulation in the polarization complex with the membrane. Like Cdc42 and Par6, the membrane-associated growth-associated protein-43 (GAP-43) is also found in the growth cone in which it is crucial for remodeling the actin cytoskeleton in response to extracellular signals (Dent and Meiri, 1998). We previously reported the novel finding that, like Cdc42 and Par6, GAP-43 is tightly associated with the centrosome of cerebellar granule cells and that, in its absence, abnormally positioned centro- 


\section{PO}
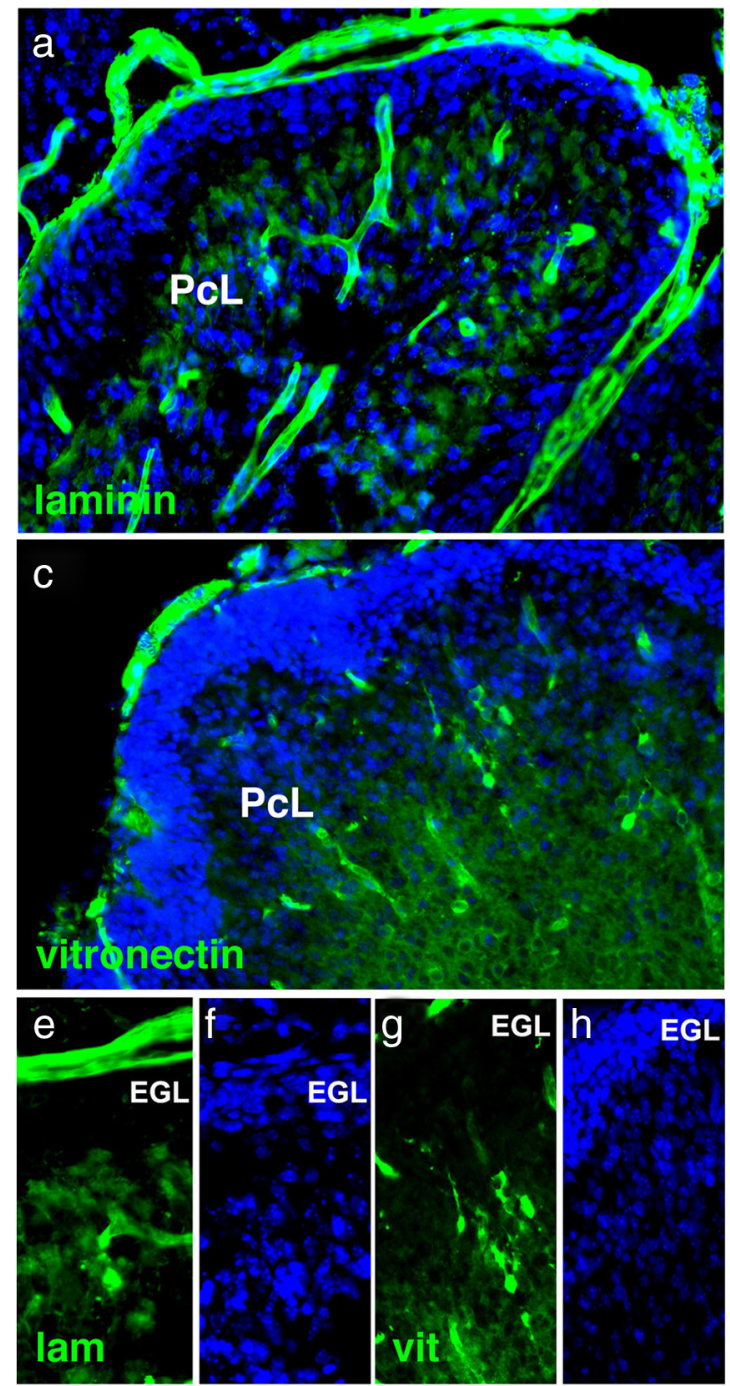

P8
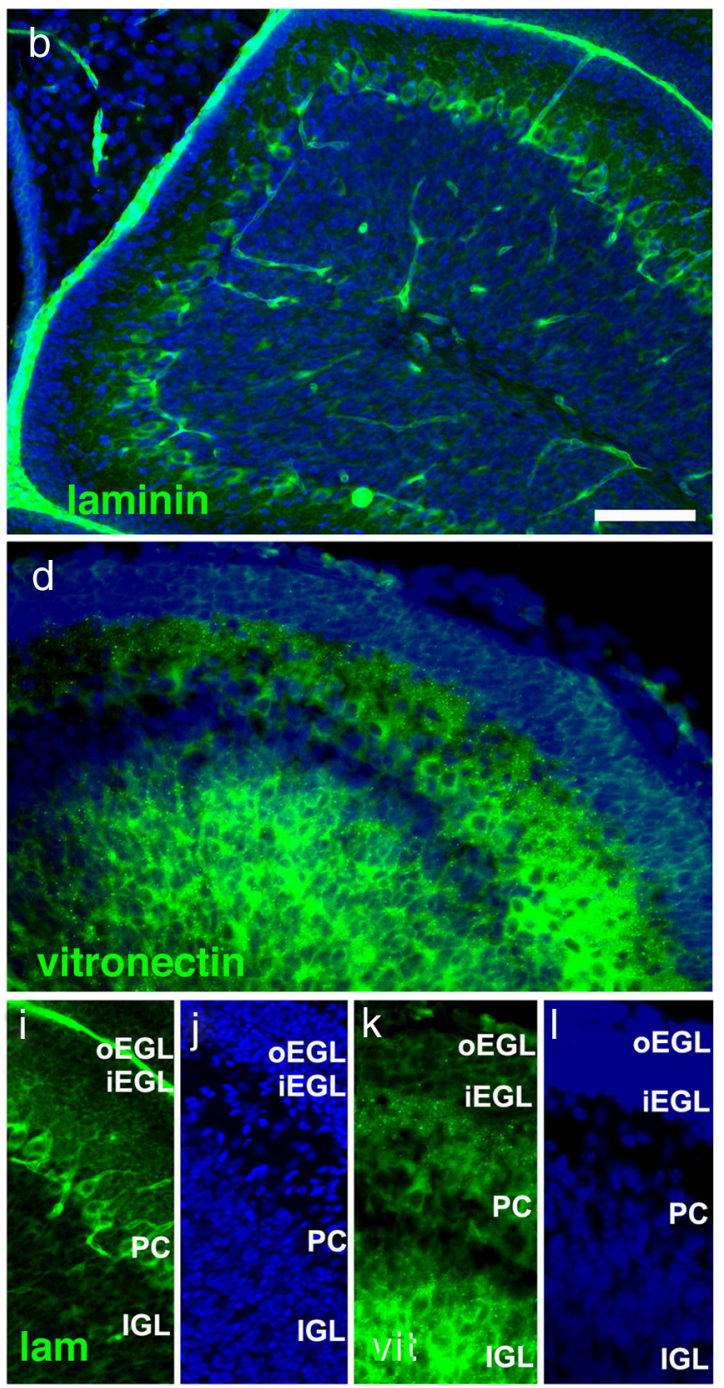

Figure 1. Laminin and vitronectin expression in mouse cerebellar cortex. Left, Sagittal section of PO cerebellum showing laminin $(\boldsymbol{a}, \boldsymbol{e})(\mathrm{lam})$ and vitronectin $(\boldsymbol{c}, \boldsymbol{g})(\mathrm{vit})$ immunoreactivity in green and DAPI labeling of nuclei for identification of cerebellar layers in blue. Right, Sagittal section of P8 cerebellum showing laminin $(\boldsymbol{b}, \boldsymbol{i})$ and vitronectin $(\boldsymbol{d}, \boldsymbol{k})$ immunoreactivity in green and DAPI labeling of nuclei for identification of cerebellar layers in blue. oEGL, outer EGL; iEGL, inner EGL. Scale bar: $\boldsymbol{a}-\boldsymbol{c}, 50 \mu \mathrm{m} ; \boldsymbol{d}, 25 \mu \mathrm{m}$.

somes are associated with disruptions to both actin and tubulin polymerization (Mishra et al., 2008). We have developed a micropatterning assay able to spatially deliver extrinsic cues to granule cells to ask whether it regulates positioning of the centrosome and how this is coordinated with intrinsic responses. We further investigated the underlying mechanism, particularly the role of GAP-43 as a coordinator between extrinsically mediated signaling and intrinsically organized responses via microtubule organization.

\section{Materials and Methods}

Animals. GAP-43-deficient mice were generated from targeted CJ7 embryonic stem cells in isogenic 129S3/imJ mice (genetic designation + Mgf-SIJ; The Jackson Laboratory stock number 002448) and backcrossed for 8-12 generations with C57BL/66N. Homozygotes $(-/-)$ mice were identified by PCR genotyping as described previously (Maier et al., 1999). Mice were maintained in specific pathogen-free conditions according to National Institutes of Health and Institutional Animal Ethical Committee (National Brain Research Centre) guidelines. The morning when the vaginal plug was detected was defined as embryonic day 0.5 and the day of birth as postnatal day $0(\mathrm{P} 0)$.
Chemicals and antibodies. Sonic hedgehog (Shh), vitronectin, and laminin were from R \& D Systems. The laminin receptor blocking antibody $\alpha 6 \beta 1$ was from Abcam, and the vitronectin receptor blocking antibody anti-integrin $\alpha 5 \beta 3$ was from Millipore Bioscience Research Reagents. The anti-integrin $\alpha 5 \beta 3$ anti-Shh blocking antibodies were from Sigma-Aldrich. Effectene transfection reagent was from Qiagen. All media and supplements were from Invitrogen. Other chemicals were from Sigma-Aldrich.

For immunostaining, anti-Shh, anti-Tau, anti-AKT, anti-phosphorylated AKT (pAKT), anti-phosphoinositide-3 kinase (PI3K), anti-tubulin, anti-IQ motif containing GTPase activating protein (IQGAP), and antivitronectin antibodies were from Santa Cruz Biotechnology. AntiCdc42, anti-patched, anti-smoothened, anti-mitogen-activated protein 2 (MAP2), and anti-Par6 antibodies were from Abcam. Anti-laminin, anti-5-bromo-2-deoxyuridine (BrdU), and anti- $\gamma$-tubulin were from Sigma-Aldrich, and anti-phosphatidylinositol 4,5-bisphosphate (PIP2) and anti-phosphatidylinositol 3,4,5-trisphosphate (PIP3) were from Echelon Biosciences. Antibodies for GAP-43 and pGAP-43 were from the Meiri laboratory. All secondary antibodies and Vectashield mounting media with 4',6'-diamidino-2-phenylindole (DAPI) were from Vector Laboratories. 
A

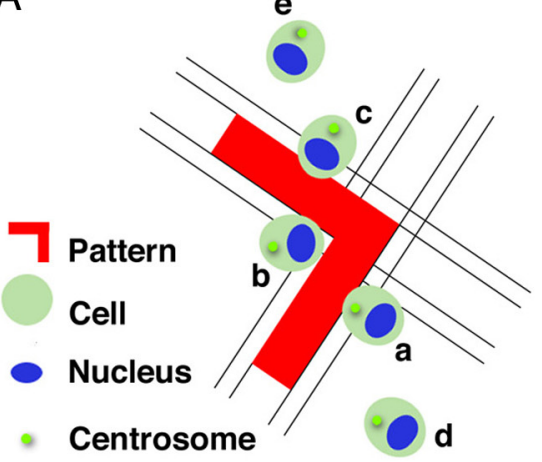

B
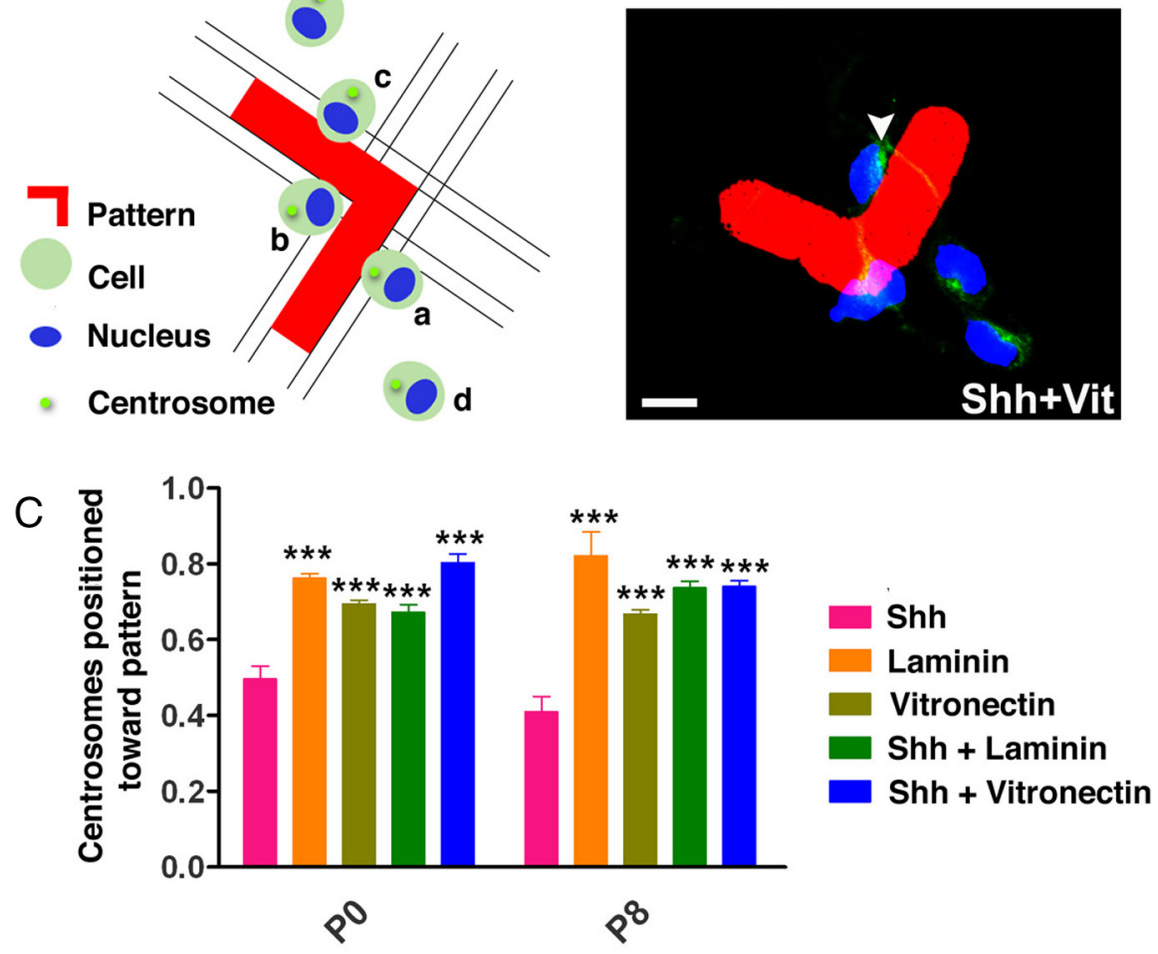

D
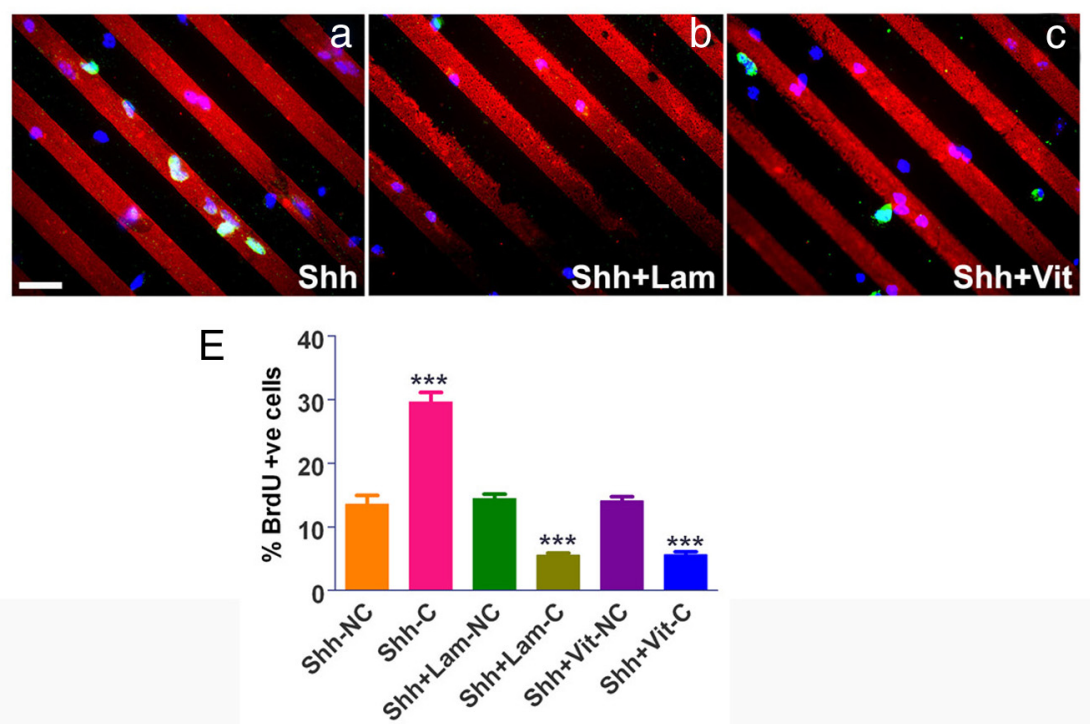

Figure 2. Laminin (Lam) and vitronectin (Vit) but not Shh can position the centrosome. $A$, Schema of centrosome orientation analysis. The central red " $\mathrm{L}$ " shape represents one printed protein pattern. $\boldsymbol{a}-\boldsymbol{c}$, Contact cells. $\boldsymbol{a}$, Centrosome positioned toward the pattern; $\boldsymbol{b}, \boldsymbol{c}$, centrosome not positioned toward the pattern. $\boldsymbol{d}, \boldsymbol{e}$, Noncontact cells. $\boldsymbol{d}$, Centrosome positioned toward the pattern; $\boldsymbol{e}$, centrosome not positioned toward the pattern. $\boldsymbol{B}$, Immunoreactivity of cells plated on vitronectin pattern (red) showing $\gamma$-tubulin labeling of centrosome (green). Arrowhead depicts contact cell with centrosome positioned toward pattern. Scale bar, $8 \mu \mathrm{m}$. C, Proportion of P0 and P8 granule cells with centrosomes positioned toward Shh, laminin, vitronectin, Shh plus laminin, and Shh plus vitronectin patterns. Data are mean \pm SEM from five independent experiments ( $\geq 400$ cells were counted in each experiment). ${ }^{* * *} p<0.001$ indicates significant difference compared with the Shh condition within each age (one-way ANOVA with post hoc Dunnett's test). Variation attributable to age was not significant (two-way ANOVA). D, Immunoreactivity of proliferating cells labeled with BrdU (green) on Shh (a), Shh plus laminin (b), and Shh + vitronectin printed patterns (c) (red). Scale bar, $25 \mu \mathrm{m}$. E, Percentage of proliferating contact cells (C) and noncontact cells (NC) on Shh, Shh plus laminin, and Shh plus vitronectin printed patterns. Data are mean \pm SEM from three independent experiments ( $\geq 200$ cells were counted in each experiment). ${ }^{* * *} p<0.001$ indicates significant difference between BrdU-positive contact and noncontact cells under each condition (one-way ANOVA with post hoc Dunnett's test).
Microcontact printing. Lithographically fabricated silicon masters were generously provided by Prof. David Juncker (McGill University, Montreal, Quebec, Canada). The stamps were made from Sylgard 184 using SU-88 as a photoresist. (Dow Corning). Proteins were printed on polyD-lysine (PDL)-coated coverslips at $10 \mu \mathrm{g} / \mathrm{ml}$ alone or in combination (Mishra et al., 2008).

Enriched cerebellar granule cell culture. Dissociated enriched granule cell cultures were established from P0 wild-type, P0 knock-out, and P8 wild-type mouse cerebellum as described previously (Gao et al., 1991). In brief, individual cerebella from $\mathrm{P} 0$ and $\mathrm{P} 8$ mice were dissected in $\mathrm{Ca}^{2+}$ and $\mathrm{Mg}^{2+}$-free Tyrode's solution and then dissociated by gentle trituration with a fire-polished glass pipette. Cultures were enriched for granule cell progenitors by removing glial contamination (Kenney et al., 2003) and plated onto poly-D-lysine $(500 \mu \mathrm{g} /$ $\mathrm{ml}$ )-coated coverslips in serum (10\%)-containing media for $24 \mathrm{~h}$ to allow the cells to recover. After $24 \mathrm{~h}$, the media were replaced with serum-free media containing DMEM/F-12 with N2 and B27 supplement (Invitrogen) with antibiotics. Cells were fixed with $4 \%$ paraformaldehyde in microtubule stabilization buffer (Zmuda and Rivas, 1998) after another $24 \mathrm{~h}$ of culture. When anti-vitronectin receptor blocking antibody $\alpha 5 \beta 3$, anti-laminin receptor blocking antibody $\alpha 6 \beta 1$, Shh blocking antibody $(10 \mu \mathrm{l} / \mathrm{ml})$, cyclopamine ( $5 \mathrm{~nm})$, bisindolylmaliemide (500 nM), wortmannin (200 nM) (Sigma-Aldrich), PIK $90(1 \mu \mathrm{M})$ (Axon Medchem), and Gö 6983 (2-[1-(3-dimethylaminopropyl)-5-methoxyindol-3-yl]-3-(1 Hindol-3-yl)maleimide) (1 $\mu \mathrm{M})$ (Calbiochem) were added, they were present throughout. For proliferation studies, cells were incubated with $10 \mu \mathrm{M}$ BrdU for $12 \mathrm{~h}$ in serum-containing media.

Cerebellar slice cultures. Cerebella from P8 C57BL/6 mice were removed, kept cold in HBSS and then sectioned into $200-\mu \mathrm{m}$-thick slices using a Mclwain Tissue Chopper-800 (Vibrotome-0800) (Gogolla et al., 2006a). The slices were cultured in Nalge Nunc International six-well plates with Millicell inserts (0.4 $\mu \mathrm{M}$; PICM03050; Millipore) in 50\% Basal Medium Eagle, 25\% HBSS, 25\% horse serum, 1 $\mathrm{mm}$ L-glutamine, $5 \mathrm{mg} / \mathrm{ml}$ glucose, and $1 \mathrm{~mm}$ penicillin/streptomycin (Gogolla et al., 2006b). Media $(200 \mu \mathrm{l})$ were replaced with the fresh media every second day. The slices were fixed after $3 \mathrm{~d}$ and processed for immunostaining. When anti-vitronectin, laminin or Shh antibodies ( $10 \mu \mathrm{l} / \mathrm{ml}$ media), or cyclopamine (5 $\mathrm{nM}$ ) were added, they were present throughout.

Immunostaining of tissue sections and cultures. Antibodies used were anti-Shh (1:200), antilaminin (1:100), anti-vitronectin (1:100), anti- $\gamma$ tubulin (1:400), anti-Par6 (1:100), anti-cdc42 (1: 50), anti-GAP-43 (1:500), anti-pGAP-43 (1:500), anti-patched (1:250), anti-smoothened (1:250), anti- $\alpha 6 \beta 1$ (1:50), anti- $\alpha 5 \beta 3$ (1:80), anti-PIP2 (1:100), anti-PIP3 (1:100), anti-PI3K (1:100), anti-MAP2 (1:500), anti-Tau (1:100), and antiIQGAP (1:100). To detect BrdU immunoreactivity, cells were treated with $4 \mathrm{~N} \mathrm{HCl}$ for $4 \mathrm{~min}$ 
to denature the DNA, followed by sodium borate neutralization $(0.1 \mathrm{M}$, $\mathrm{pH} 8.5$ ).

Immunostaining of cerebellar slice cultures. Immunostaining of cerebellar slices was performed according to published protocol (Gogolla et al., 2006b). Slices were fixed in $4 \%$ paraformaldehyde, after which they were incubated with $20 \%$ methanol/PBS solution for 5 min. Triton X-100 solution at $1 \%$ for $12 \mathrm{~h}$ at $4^{\circ} \mathrm{C}$ was used to permeablize the slices. Slices were incubated with primary antibody overnight at $4^{\circ} \mathrm{C}$. Incubation with secondary antibody was done at room temperature for $3 \mathrm{~h}$.

Centrosome positioning analysis in dissociated cells in vitro. Enriched granule cell cultures were plated on microcontact printed coverslips, fixed and immunostained to detect printed protein and the centrosome. As depicted in Figure $2 \mathrm{~A}$, cells touching the microcontact pattern were designated "contact" cells, whereas cells not touching the pattern were designated "noncontact" cells. Only single cells were included in the analysis. To assess the position of the centrosome relative to the microcontact pattern, a line parallel to each arm of the pattern and passing through the middle of the cells was drawn, and the position of the centrosome with respect to the parallel midline was analyzed. Centrosomes positioned in the proximal half of the cell touching the pattern were classified as oriented toward the pattern, whereas those positioned in the distal half of the cell were classified as not oriented toward the pattern. For each field, the position of all contact and noncontact cells were analyzed. The total number of cells counted for all combinations of microcontact printed proteins is summarized in supplemental Table S1 (available at www.jneurosci.org as supplemental material).

Centrosome positioning analysis in cerebellar slice cultures. Confocal images of slices were taken with a Carl Zeiss LSM 510 Meta microscope and offline analysis was performed using NIH Image J software. From each image, three random areas of $625 \mu \mathrm{m}^{2}$ were identified, and the orientation of centrosome in each was analyzed for each of the cells and classified in two types as follows: type I cells, in which the centrosome was positioned at the base of the leading process, and type II cells, in which the centrosome was not positioned at the base of the leading process.

Protein analysis. Cells were plated in $60 \mathrm{~mm}$ dishes at a density of $2 \times$ $10^{6}$ cells $/ \mathrm{cm}^{2}$ and collected into lysis buffer $[50 \mathrm{Mm}$ Tris-Cl, $\mathrm{pH} 7.5,150$ mм NaCl, 1 mм EDTA, 1 mм EGTA, 50 mm sodium fluoride, $1 \mathrm{~mm}$ sodium orthovanadate, $2 \%$ SDS, and protease inhibitor mixture] after $48 \mathrm{~h}$. Western blotting was performed according to Sharma et al. (2003). Protein levels were quantified using Chemi Genius2 software (SynGene) to obtain integrated density values.

Phospholipid binding. GAP-43 was purified from P0 rat brains using reverse-phase HPLC (Changelian et al., 1990) and used to probe PIP strips (Echelon Biosciences) according to the instructions of the manufacturer. Bound total GAP-43 was detected with the 7B10 monoclonal antibody, and bound PKC-phosphorylated GAP-43 (pGAP-43) was detected with the 2 G12 monoclonal antibody. Immunoreactivity was revealed by chemiluminescence, and the film was exposed within the linear range of the signal. The experiment was repeated three times using different preparations of purified GAP-43, and results were quantitated by densitometry, as above.

GAP-43 short hairpin RNA. The mU6 promoter and GAP-43 short hairpin RNA (shRNA) constructs were cloned into the enhanced green fluorescent protein (EGFP)-Simian virus 40-phosphorylated cytomegalovirus (pCMV) vector and verified by PCR and sequencing (Mishra et al., 2008). The plasmid vectors pCMV-EGFP (mock) and pCMVshRNA plasmid DNA constructs were used for transfection of granule cells after $24 \mathrm{~h}$ of culture using $0.5 \mu \mathrm{g}$ of plasmid DNA, $4 \mu \mathrm{l}$ of enhancer (R), and $10 \mu \mathrm{l}$ of Effectene reagent according to the directions of the manufacturer. Media were changed after $6 \mathrm{~h}$ of incubation, and cells were fixed with $4 \%$ paraformaldehyde after $48 \mathrm{~h}$ of transfection.

Statistical analysis. The data are expressed as mean \pm SEM. Data were analyzed with one-way ANOVA (GraphPad Prism version 5 for Windows; GraphPad Software). When ANOVA showed significant differences among multiple experimental groups, multiple comparisons between experimental groups were performed using Dunnett's or Bonferroni's post hoc test.
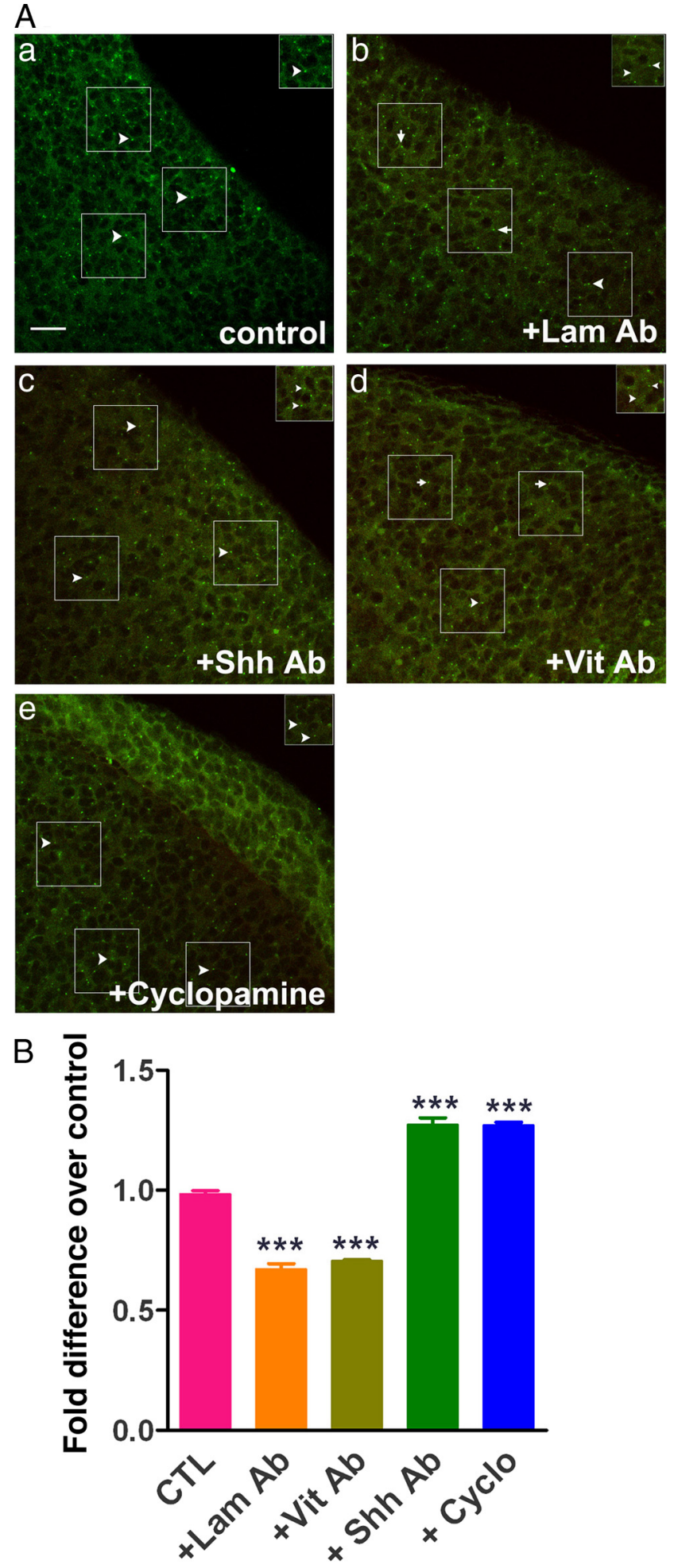

Figure 3. Inhibiting laminin (Lam) and vitronectin (Vit) prevents centrosome positioning in slice cultures. $\boldsymbol{A}$, Confocal micrographs of P8 cerebellar slice cultures labeled with anti- $\gamma$ tubulin to detect centrosomes (green). $\boldsymbol{a}$, Control. $\boldsymbol{b}-\boldsymbol{d}$, In the presence of blocking antibodies $(\mathrm{Ab})$ : $\boldsymbol{b}$, anti-laminin; $\boldsymbol{c}$, anti-Shh; $\boldsymbol{d}$, anti-vitronectin. $\boldsymbol{e}$, In the presence of $5 \mathrm{~nm}$ cyclopamine (Cyclo). Arrowheads, Type I cells with centrosomes positioned at the base of the primary axon. Arrows, Type ll cells with centrosomes not positioned at the base of the primary axon. Scale bar, $25 \mu \mathrm{m}$. B , Proportion of type I cells compared with control (CTL) for each condition. Data are mean \pm SEM from $\geq 3$ independent experiments ( $\geq 200$ cells were counted in each experiment). ${ }^{* *} p<0.001$ indicates significant differences between treated and control cells (oneway ANOVA with post hoc Dunnett's test). 


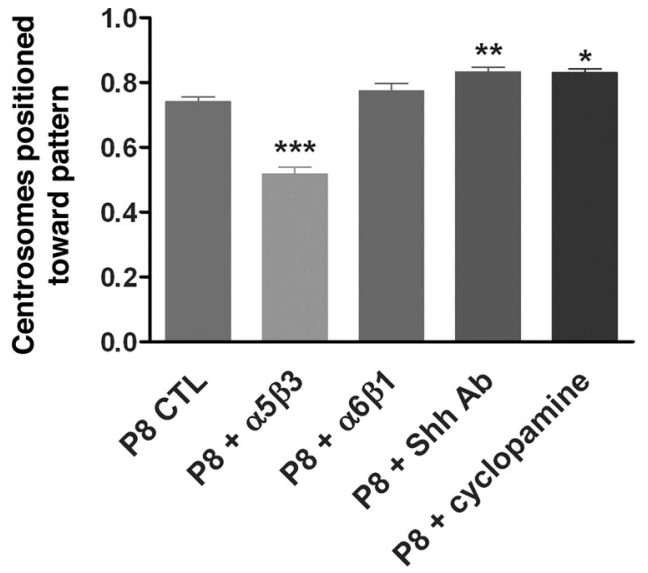

Figure 4. Laminin and vitronectin require cognate integrin receptors to position the centrosome. Proportion of P8 granule cells with centrosomes positioned toward Shh plus vitronectin protein pattern (P8 CTL) or in presence of anti-vitronectin receptor antibody anti- $\alpha 5 \beta 3$ (P8 + $\alpha 5 \beta 3$ ), anti-laminin receptor antibody anti- $\alpha 6 \beta 1$ (P8 $+\alpha 6 \beta 1)$, anti-Shh antibody (P8 + Shh Ab), or cyclopamine (P8 + cyclopamine). Data are mean \pm SEM of $\geq 4$ independent experiments ( $\geq 400$ cells were counted in each experiment). ${ }^{*} p<0.05,{ }^{* *} p<0.01$, and ${ }^{* * *} p<0.001$ indicate significant differences compared with control (one-way ANOVA with post hoc Dunnett's test)

\section{Results}

Laminin and vitronectin are differentially expressed in the cerebellum during the first postnatal week

Laminin and vitronectin were differentially localized in the developing cerebellum in vivo at both P0 and P8. Figure 1 shows that, at $\mathrm{P} 0$, high levels of laminin were found in the pia and the area that will become the Purkinje cell layer (PcL). Conversely, levels of vitronectin were lower in the PcL, and strong expression was confined to the deeper layers of the cerebellum. By P8, laminin immunoreactivity extended into the developing molecular layer and the deeper layers of the cerebellum, whereas levels in the outer external granule layer (EGL) remained low. Vitronectin was also expressed in the PcL and its arborization in the molecular layer but was more strongly upregulated in the EGL and in deeper layers of the cerebellum including the inner granule layer (IGL) compared with laminin. In contrast, the integrin receptors for laminin and vitronectin $(\alpha 6 \beta 1$ and $\alpha 5 \beta 3)$ were more diffusely localized at both P0 and P8 (data not shown). The results show that laminin and vitronectin are differentially localized in the developing cerebellum in vivo, consistent with a role in influencing polarization of granule cell precursors as they initiate migration from the EGL through the molecular layer.

\section{Both laminin and vitronectin can induce centrosome positioning in vitro}

To assess whether the differential distribution of laminin and/or vitronectin reflects roles as a spatially restricted extrinsic cue to regulate positioning of centrosomes, we developed an in vitro micropatterning assay in which cultures enriched in granule cell precursors were plated onto coverslips on which patterns of either vitronectin or laminin with or without Shh had been laid down by microcontact printing, and centrosome positioning with respect to the printed protein pattern was evaluated. To ensure that equivalent amount of Shh and the extracellular matrix (ECM) molecules were present in micropatterns containing more than one printed, the micropatterns were immunostained for the presence of both constituents (supplemental Fig. S1, available at www.jneurosci.org as supplemental material). Figure $2 \mathrm{~A}$
A

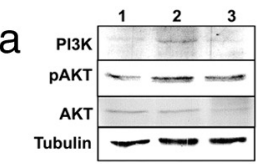

b

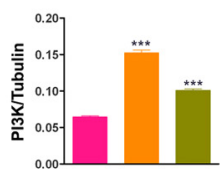

C

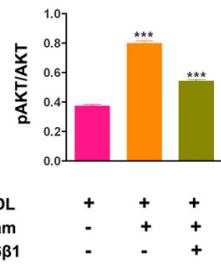

C

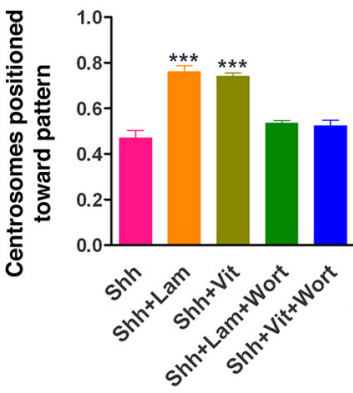

$E$

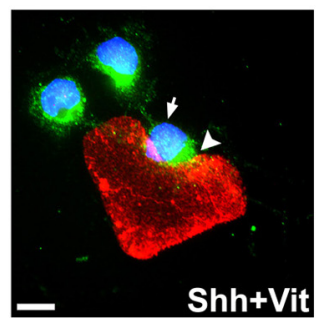

B a

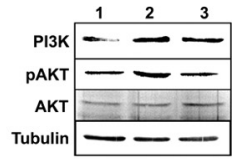

b

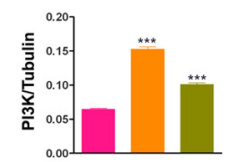

C
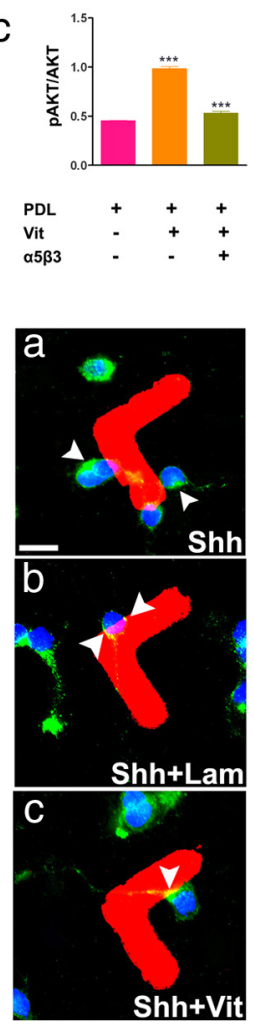

Figure 5. Laminin and vitronectin require upregulation of PI3K to position the centrosome. $\boldsymbol{A}$, Influence of laminin (Lam). $\boldsymbol{a}$, Representative Western blot from P8 granule cells after $48 \mathrm{~h}$ of culture probed with anti-PI3K, PAKT, AKT, and tubulin. Lane 1, PDL; lane 2, PDL plus laminin; lane 3, PDL plus laminin in the presence of laminin receptor blocking antibody $\alpha 6 \beta 1 . \boldsymbol{b}$, Quantitation of PI3K immunoreactivity (normalized relative optical densities) compared with total tubulin. c, Quantitation of pAKT immunoreactivity normalized to total AKT. Data are mean \pm SEM from four independent experiments. ${ }^{* * *} p<$ 0.001 indicates significant difference compared with control (PDL) (one-way ANOVA with post hoc Dunnett's test). $\boldsymbol{B}$, Influence of vitronectin (Vit). $\boldsymbol{a}$, Representative Western blot from P8 granule cells after $48 \mathrm{~h}$ of culture probed with anti-PI3K, pAKT, AKT, and tubulin. Lane 1, PDL; lane 2, PDL plus vitronectin; lane 3, PDL plus vitronectin in the presence of vitronectin receptor blocking antibody $\alpha 5 \beta 3$. $\boldsymbol{b}$, Quantitation of PI3K immunoreactivity (normalized relative optical densities) compared with tubulin. c, Quantitation of pAKT immunoreactivity normalized to total AKT. Data are mean \pm SEM from four independent experiments. ${ }^{* *} p<0.001$ indicates significant difference compared with control (PDL) (one-way ANOVA with post hoc Dunnett's test). C, Proportion of P8 granule cells with centrosomes positioned toward Shh, Shh plus laminin, Shh plus vitronectin, Shh plus laminin with $200 \mathrm{~nm}$ wortmanin (Wort), and Shh plus vitronectin with $200 \mathrm{~nm}$ wortmanin. Data are mean \pm SEM of $\geq 4$ independent experiments ( $\geq 400$ cells were counted in each experiment). ${ }^{* *} p<0.001$ indicates significant differences compared with control (oneway ANOVA with post hoc Dunnett's test). D, PI3K (green) immunoreactivity (arrowheads) in PO cerebellar granule cells plated on Shh (a) (red), Shh plus laminin (b), and Shh plus vitronectin (c) microcontact printed patterns. Scale bar, $8 \mu \mathrm{m}$. E, PIP3 (green) immunoreactivity in $\mathrm{PO}$ cerebellar granule cells plated on Shh plus vitronectin microcontact printed pattern. Arrowhead indicates PIP3 immunoreactivity, whereas arrow indicates region of minimal PIP3 immunoreactivity. Scale bar, $8 \mu \mathrm{m}$. 
A
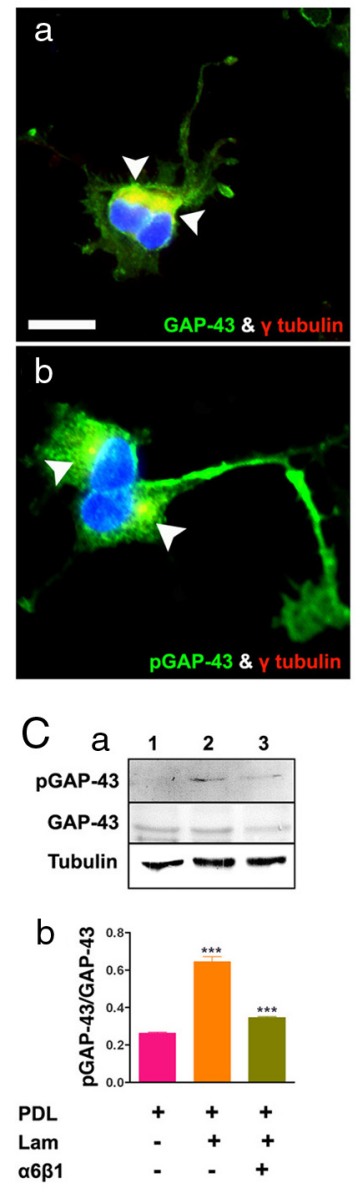

B
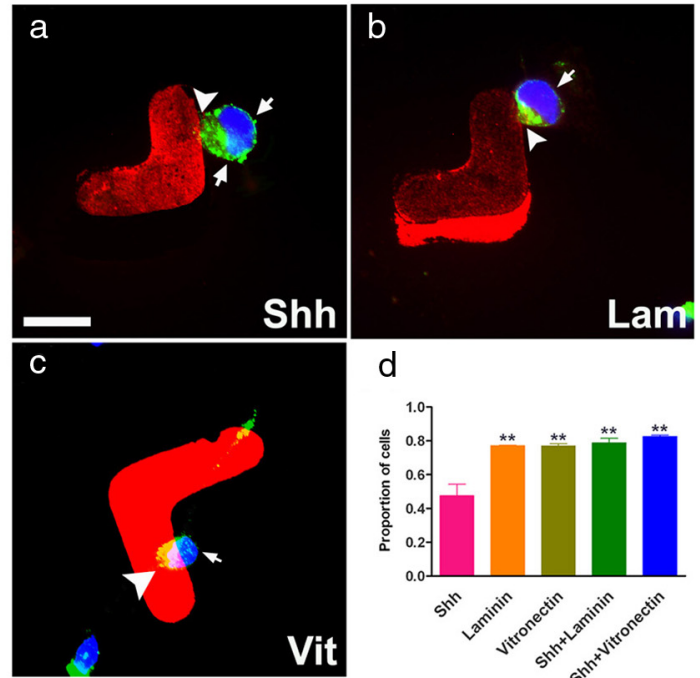

d
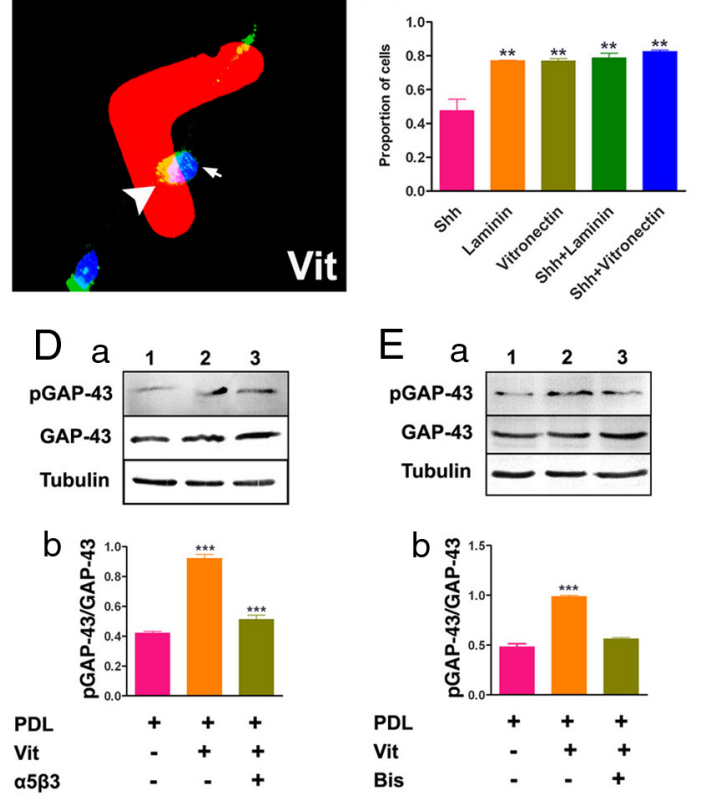

E
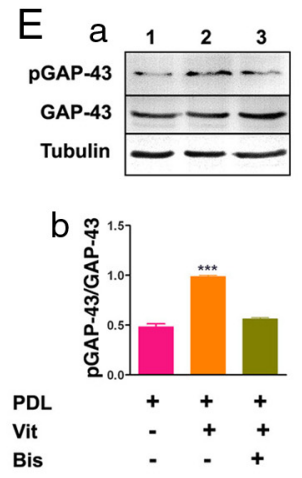

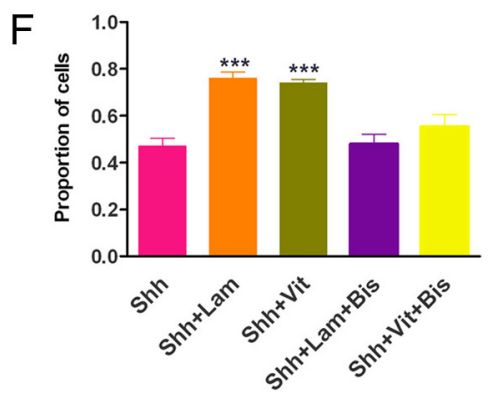

Figure 6. Laminin (Lam) and vitronectin (Vit) require phosphorylated GAP-43 to position the centrosome. $\boldsymbol{A}, \mathrm{P} 0$ granule neurons labeled with GAP-43 (green) and $\gamma$-tubulin (red) (a) and labeled with pGAP-43 (green) and $\gamma$-tubulin (red) (b). Arrowheads shows region in which GAP-43 and pGAP-43 are coexpressed with $\gamma$-tubulin. Scale bar, $8 \mu \mathrm{m}$. B, GAP-43 (green) immunoreactivity in P0 cerebellar granule cells plated on Shh $(\boldsymbol{a})$ (red), laminin (b) (red), and vitronectin (c) (red) microcontact printed patterns. Arrowheads indicate pGAP-43 expression in the region of cell contact, whereas arrow indicates pGAP-43 expression in the region not in contact. Scale bar, $8 \mu \mathrm{m}$. d, Proportion of cells with pGAP-43 immunoreactivity positioned toward Shh, laminin, Shh plus laminin, vitronectin, and Shh plus vitronectin protein patterns. Data are mean \pm SEM from three independent experiments ( $\geq 100$ cells were counted in each experiment). ${ }^{* *} p<0.01$ indicates significant difference in $\mathrm{PGAP}-43$ localization compared with Shh values (one-way ANOVA with post hoc Dunnett's test). $\boldsymbol{C}$, Influence of laminin. $\boldsymbol{a}$, Representative Western blot from P8 granule cells after $48 \mathrm{~h}$ of culture probed with anti-pGAP-43, GAP-43, and tubulin. Lane 1, PDL; lane 2, PDL plus laminin; lane 3, PDL plus laminin in the presence of laminin receptor blocking antibody anti- $\alpha 6 \beta 1$. $\boldsymbol{b}$, Quantitation of pGAP-43 immunoreactivity (normalized relative optical densities) compared with GAP-43. Data are mean \pm SEM from four independent experiments. ${ }^{* * *} p<0.001$ indicates significant difference compared with control (PDL) (one-way ANOVA with post hoc Dunnett's test). $\boldsymbol{D}$, Influence of vitronectin. $\boldsymbol{a}$, Representative Western blot from P8 granule cells after $48 \mathrm{~h}$ of culture probed with anti-pGAP-43, GAP-43, and tubulin. Lane 1, PDL; lane 2, PDL plus vitronectin; lane 3, PDL plus vitronectin in the presence of laminin receptor blocking antibody anti- $\alpha 5 \beta 3 . \boldsymbol{b}$, Quantitation of pGAP-43 immunoreactivity (normalized relative optical densities) compared with GAP-43. Data are mean \pm SEM from four independent experiments. ${ }^{* * *} p<0.001$ indicates significant difference compared with control (PDL) (one-way ANOVA with post hoc Dunnett's test). E, PKC inhibition. Representative Western blot from P8 granule cells after $48 \mathrm{~h}$ of culture probed with anti-pGAP-43, GAP-43, and tubulin (a). Lane 1, PDL; lane 2, PDL plus vitronectin; lane 3, PDL in the presence of $500 \mathrm{~nm}$ bisindolylmaleimide (Bis.). $\boldsymbol{b}$, Quantitation of pGAP-43 immunoreactivity (normalized relative optical densities) compared with GAP-43. Data are mean \pm SEM from four independent experiments. ${ }^{* * *} p<0.001$ indicates significant

depicts a schematic of how centrosome orientation was analyzed. [Details are provided in Materials and Methods, and details of the number of cells included in the analysis are summarized in supplemental Table S1 (available at www. jneurosci.org as supplemental material).] The central red " $\mathrm{L}$ " shape represents one printed protein pattern. Cells labeled a-c are in contact with the pattern, but, whereas the centrosome in cell a is positioned toward the pattern, centrosomes in cells $b$ and $c$ are not. Cells labeled $d$ and e are not in contact with the pattern, but, whereas the centrosome in cell $\mathrm{d}$ is positioned toward the pattern, the centrosome in cell e is not. Figure $2 B$ shows a representative image of centrosome position, detected with anti- $\gamma$-tubulin immunoreactivity, when granule cell precursors were plated on a vitronectin plus Shh patterned coverslip. The arrowhead indicates a contact cell in which the centrosome is positioned toward the pattern. Figure $2 C$ shows that the centrosomes in granule cell precursors that contacted a pattern as a result of Shh alone were not positioned toward the pattern, whereas centrosomes in cells contacting a pattern as a result of either laminin or vitronectin were positioned toward the protein patterns, whether or not Shh was present. The results indicate that centrosome position is affected by the presence of laminin and vitronectin, not by Shh.

\section{Both laminin and vitronectin inhibit} the ability of sonic hedgehog to act as a mitogen for cerebellar granule cell precursors

Developing cerebellar granule cell precursors proliferate under the regulation of Shh, and previous results have shown that, although vitronectin can inhibit Shhmediated mitogenicity, thereby inducing differentiation, laminin substrates potentiate mitogenicity of soluble Shh (Pons et al., 2001). Because Shh is thought to interact with the ECM directly (Pons and Martí, 2000; Pons et al., 2001), we used our micropatterning assay to examine how incorporating Shh into laminin and

$\leftarrow$

difference compared to control (PDL) (one-way ANOVA with post hoc Dunnett's test). $\boldsymbol{F}$, Proportion of $\mathrm{P} 8$ granule cells with centrosomes positioned toward Shh, Shh plus laminin, Shh plus vitronectin, Shh plus laminin with $500 \mathrm{~nm}$ bisindolylmaleimide, and Shh plus vitronectin with $500 \mathrm{~nm}$ bisindolylmaleimide. Data are mean \pm SEM of $\geq 4$ independent experiments ( $\geq 400$ cells were counted in each experiment). ${ }^{* * *} p<0.001$ indicates significant differences compared with Shh (one-way ANOVA with post hoc Dunnett's test). 
A
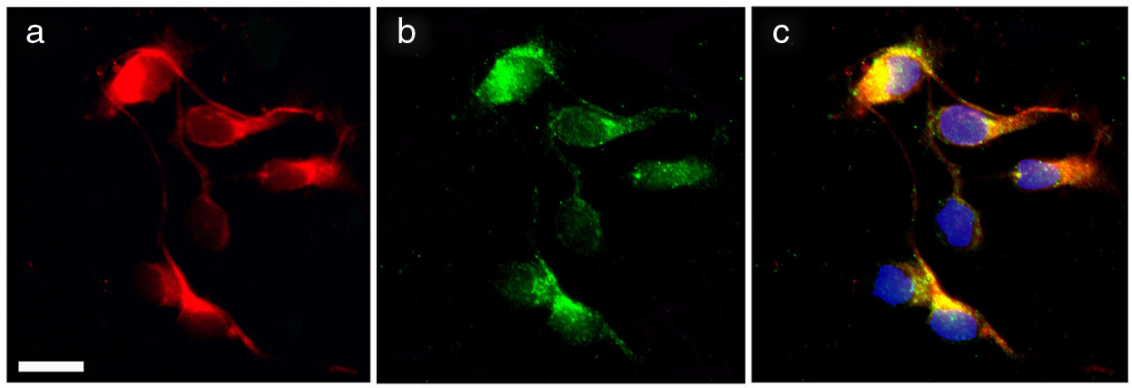

B

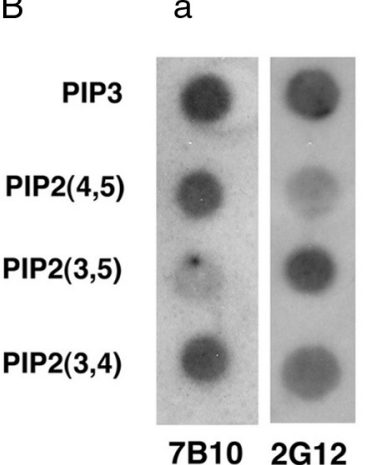

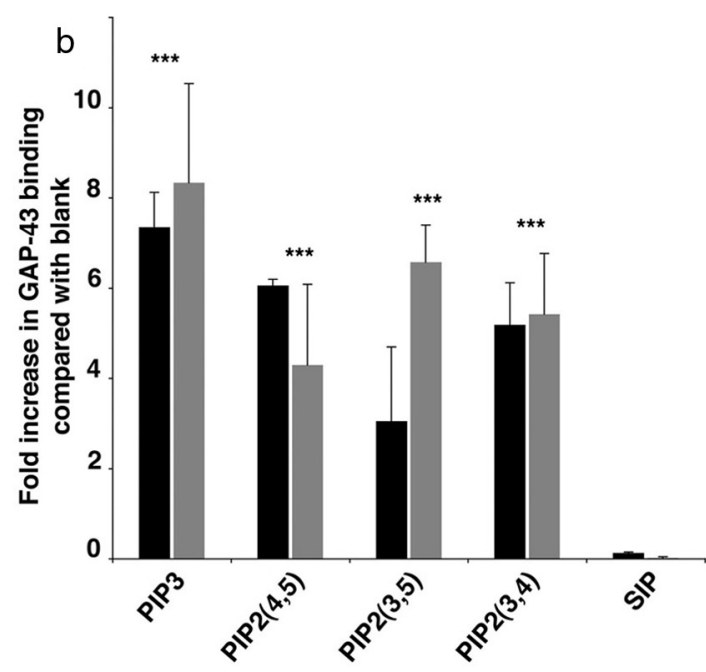

Figure 7. Phosphorylated GAP-43 interacts with PIP2 and PIP3. $\boldsymbol{A}$, P8 granule cells labeled with GAP-43 (a) (red) and PIP3 (b) (green). c, Merged images to show colocalization. Scale bar, $8 \mu \mathrm{m}$. B, Phospholipid binding. $\boldsymbol{a}$, Membrane phospholipids incubated with pure GAP-43 and then probed with antibodies that recognize total GAP-43 (7B10) and pGAP-43 (2G12). $\boldsymbol{b}$, Quantitation of the fold increase in GAP-43 (black bar) and pGAP-43 (gray bar) immunoreactivity (normalized relative optical densities) compared with blank control. Data are mean \pm SEM from three $(n=3)$ independent experiments. ${ }^{* *} p<0.001$ indicates significant differences compared with Sphingosine-1-phosphate (SIP) (one-way ANOVA with post hoc Dunnett's test).

whereas significantly fewer centrosomes remained aligned after the slices had been treated with either laminin or vitronectin antibodies. In contrast, the percentage of centrosomes aligned with the leading process significantly increased after the slices had been treated with either Shh blocking antibodies or cyclopamine. The results are therefore consistent with the data from the micropatterning assay and confirm that it provides an appropriate strategy with which to examine laminin and vitronectin function in centrosome positioning during polarization.

Centrosome positioning depends on signaling through laminin and vitronectin integrin receptors Laminin and vitronectin signal directly through the $\alpha 6 \beta 1$ and $\alpha 5 \beta 3$ integrin receptors, respectively, whereas Shh uses the patched receptor (Martí and Bovolenta, 2002). We therefore used cognate integrin blocking antibodies to assess whether they inhibited centrosome positioning in the micropatterning assay. In the presence of antibody that recognizes the laminin integrin receptor $\alpha 6 \beta 1$, the proportion of contact cells with centrosomes positioned toward the laminin pattern was significantly reduced $(0.48 \pm 0.03$ compared with $0.82 \pm 0.02 ; p<0.001$ by unpaired $t$ test). In the presence of antibody that recognizes the vitronectin integrin receptor $\alpha 5 \beta 3$, the proportion of contact cells with centrosomes positioned toward the vitronectin pattern was likewise significantly reduced $(0.48 \pm 0.04$ compared with

vitronectin micropatterns affected its mitogenic properties. Figure 2, $D$ and $E$, shows that Shh incorporated into a micropattern could still induce proliferation of P0 granule cell, as detected with BrdU labeling. However, when Shh was combined with either laminin or vitronectin, the number of contact cells labeled with BrdU was significantly reduced compared with cells contacting Shh alone or cells contacting PDL control substrate (Fig. 2 E, NC lanes). The results, quantitated in Figure $2 E$, show that, under these conditions, laminin as well as vitronectin inhibits the mitogenic activity of substrate-associated Shh. Hence, both laminin and vitronectin enhanced the differentiation of granule cell precursors in our micropatterning assay.

Inhibiting laminin or vitronectin binding inhibits, whereas inhibiting Shh enhances, appropriate centrosome positioning in slice cultures

We used P8 cerebellar slice cultures as a model that most closely mimics in vivo development, to investigate whether laminin and vitronectin act as extrinsic cues to position the centrosome at the base of the leading process as migration is initiated. We incubated the slices for $48 \mathrm{~h}$ with antibodies having blocking activity against laminin, vitronectin, or Shh or with the pharmacological Shh inhibitor cyclopamine and then used anti- $\gamma$-tubulin immunoreactivity to detect the location of the centrosome with respect to the leading process as before (Fig. 3). In control slices, $70 \%$ of the centrosomes were aligned with the base of the leading process,
$0.67 \pm 0.01 ; p<0.01$ by unpaired $t$ test). In addition, Figure 4 shows that, when the $\alpha 6 \beta 1$ integrin was blocked in the presence of vitronectin, there was no effect on centrosome orientation, indicating that the interaction of the receptor with its ligand was specific. Both laminin and vitronectin can modulate Shh signaling, but neither Shh inhibitory antibodies nor cyclopamine were able to inhibit the positioning response to vitronectin, indicating that centrosome positioning requires integrin, not Shh, signaling.

\section{Centrosome positioning induced by laminin and vitronectin} requires upregulation of $\mathrm{PI} 3 \mathrm{~K}$

Activation of integrin receptors after ligand binding results in upregulation of PI3K. To determine whether laminin and vitronectin induce PI3K activation, we plated granule cells on laminin, vitronectin, or PDL for $48 \mathrm{~h}$ and then monitored PI3K activation on Western blots. Figure 5 shows that cells plated on laminin (Fig. $5 A$ ) or vitronectin (Fig. $5 B$ ) had increased levels of PI3K compared with cells plated on PDL alone. Moreover, pAKT, a downstream target of PI3K, was also upregulated on both laminin (Fig. $5 A c$ ) and vitronectin (Fig. $5 B c$ ). Upregulation of both PI3K and pAKT was inhibited in the presence of blocking antibodies against the laminin and vitronectin integrin receptors $\alpha 6 \beta 1$ or $\alpha 5 \beta 3$. We next used the PI3K inhibitor wortmannin to determine whether centrosome positioning by laminin and vitronectin require upregulation of PI3K. Figure $5 C$ shows that wortmannin inhibited centrosome positioning in response to both laminin 
and vitronectin. To confirm the involvement of the PI3K, a second PI3K inhibitor (PIK 90) was also used to inhibit centrosome orientation (supplemental Fig. S2, available at www.jneurosci.org as supplemental material). In additional support of the role of PI3K in centrosome orientation, Figure $5 D$ shows that $\mathrm{PI} 3 \mathrm{~K}$ is concentrated at the region of cell contact with the extrinsic cue on either a vitronectin or laminin pattern, whereas it is not concentrated at the region in contact with an Shh micropattern alone. Moreover, in agreement with the foregoing results that Shh does not inhibit the activity of vitronectin, PI3K remained colocalized at the contact point on a micropattern containing both Shh and vitronectin. Activation of PI3K converts PIP2 to PIP3, and so we also investigated whether PIP3 is located at the region of cell contact with the extrinsic cue. Figure $5 E$ shows that PIP3 was concentrated at the region of contact on Shh plus vitronectin, as was shown for PI3K. Together, these results indicate that centrosome orientation requires activation of the PI3K signaling pathway and show that PI3K activation is associated with PIP3 localization in which the centrosome is positioned.

\section{Centrosome positioning requires \\ PKC-phosphorylated GAP-43}

In contrast with PIP2 that can modulate actin dynamics directly, PIP3 modulates actin dynamics via its interaction with actin regulatory proteins. One actin modulatory protein that is associated with centrosomes is GAP-43. pGAP-43 is highly enriched in the EGL along with laminin and vitronectin, and we have shown previously that, when it is genetically deleted, centrosome positioning in mitosing granule cell precursors is abnormal both in vitro and in vivo (Mishra et al., 2008). Figure $6 A$ shows that, in polarizing precursors too, pGAP-43 is colocalized with centrosomes at the base of primary process. We therefore used the micropatterning assay to determine how extrinsic cues affect the distribution of pGAP-43. Figure $6 B$ shows that pGAP-43 was enriched at the region of contact with either a laminin or vitronectin with or without Shh micropattern but not with a Shh micropattern alone. Previous studies have shown that PKC-mediated GAP-43 phosphorylation can occur in culture under basal conditions (Dent and Meiri, 1992). The increase in specific activity of GAP-43 phosphorylation in response to laminin or vitronectin could be prevented by blocking cognate integrin receptors with specific receptor antibodies (Fig. 6C,D) or after treatment with bisindolylmaleimide or the PKC inhibitor Gö 6983 to inhibit PKC (Fig. 6E) (supplemental Fig. S3, available at www.jneurosci.org as supplemental material). Together with Figure $6 B$, the PKCdependent increase in GAP-43 phosphorylation in response to laminin or vitronectin seems to occur at the region of contact. Inhibiting
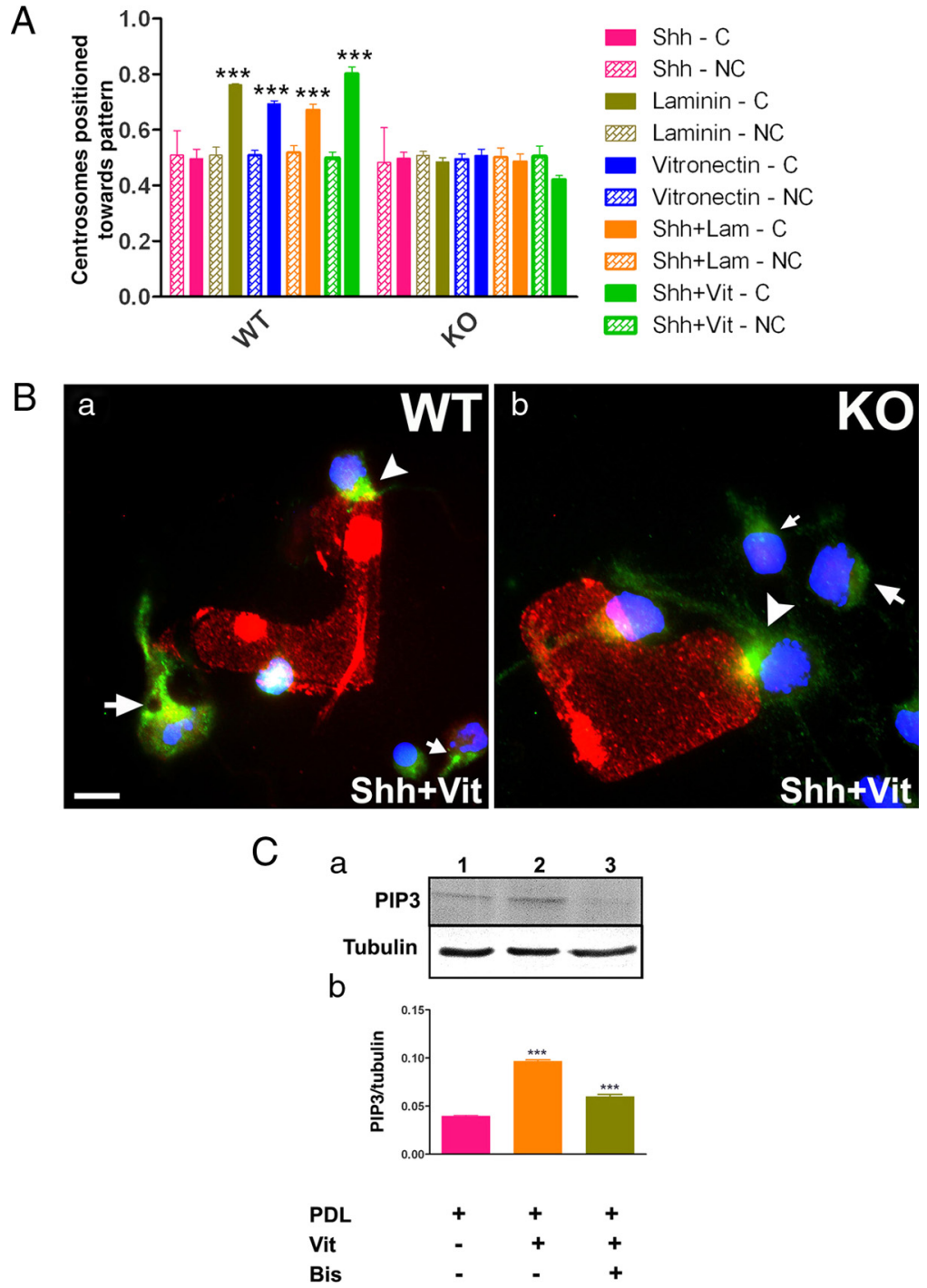

Figure 8. GAP-43 is required for positioning centrosomes but not for PIP3 localization, but PIP3 upregulation is dependent on PKC. A, Proportion of PO GAP- $43^{+/+}$and GAP- $43^{-/-}$granule cells with centrosomes positioned toward Shh, laminin, vitronectin, Shh plus laminin, Shh plus vitronectin, and Shh plus laminin protein patterns. Data are mean \pm SEM of $\geq 4$ independent experiments ( $\geq 400$ cells were counted in each experiment). ${ }^{* * *} p<0.001$ indicates significant differences compared with noncontact cell values (one-way ANOVA (red). $\boldsymbol{a}, G A P-43^{+/+} ; \boldsymbol{b}, G A P-43^{-/-}$. Arrowhead points to the region of the cell in contact with the protein pattern, and arrow points to the base of the primary process in cells not in contact with the pattern. $\boldsymbol{C}, \boldsymbol{a}$, Western blot probed with PIP3 and tubulin antibody after $48 \mathrm{~h}$ of P8 leimide (Bis.) (lane 3). $\boldsymbol{b}$, Quantitation of PIP3 normalized to total tubulin after $48 \mathrm{~h}$ of culture. Western blot shown is a representative blot. Bars in Western blot analysis represent normalized relative optical densities plotted as mean \pm SEM calculated from three $(n=3)$ independent experiments. ${ }^{* * *} p<0.001$ indicates significant difference from control (PDL) (one-way ANOVA with Dunnett's test compared with PDL values). KO, Knock-out; WT, wild type; C, contact; NC, noncontact.

PKC by either bisindolylmaleimide or Gö 6983 also prevented centrosome positioning in response to either laminin or vitronectin (Fig. 6C-F) (supplemental Fig. S2, available at www.jneurosci.org as supplemental material). The results indicate that $\mathrm{PKC}$ activation is required for laminin and vitronectin to be able to position the centrosome at the base of the primary process and that this PKC activation in turn causes phosphorylation of GAP-43 in the region in which contact occurs.

\section{PKC-phosphorylated GAP-43 interacts directly with PIP2 and PIP3}

Figure 7 shows that pGAP-43 in the region of the centrosome colocalizes with PIP3. Moreover, direct binding assays using purified 
A

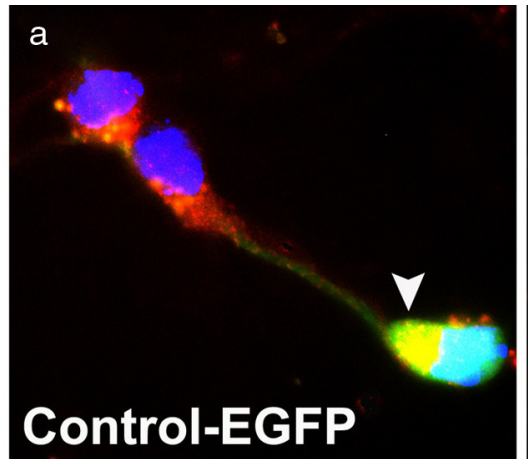

B

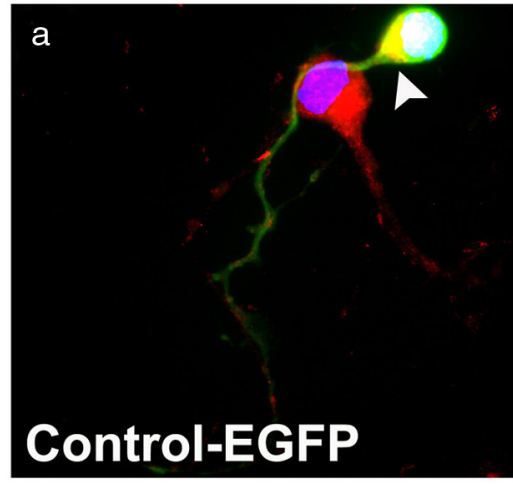

C
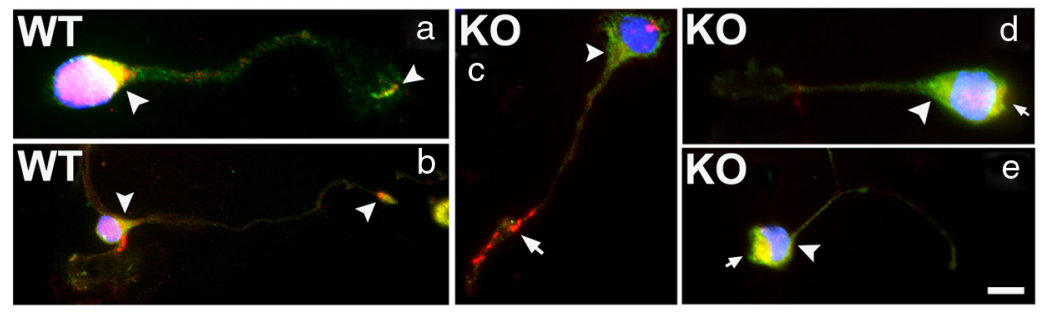

D
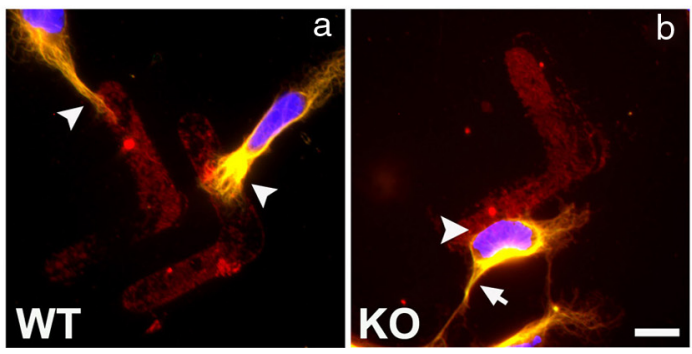

Figure 9. GAP-43 is required for localization of cytoskeletal polarity regulators and their ability to position the centrosome. $A$, Effect of acutely inhibiting GAP-43 expression on localization of Cdc42 (red) in P0 granule cells. $\boldsymbol{a}$, Control-EGFP construct (green). $\boldsymbol{b}$, GAP-43 shRNA-EGFP (green). Arrowhead points to the base of the primary process. Scale bar, $8 \mu \mathrm{m}$. B, Effect of acutely inhibiting GAP-43 expression on localization of Par6 (red) in P0 granule cells. $\boldsymbol{a}$, Control-EGFP construct (green). $\boldsymbol{b}$, GAP-43 shRNA-EGFP (green). Arrowhead points to the base of the primary process. Representative images from $n \geq 25$ cells from three independent experiments. Scale bar, $8 \mu \mathrm{m}$. C, Immunoreactivity of Cdc42 (green) and IQGAP1 (red) in P0 granule cells. $\boldsymbol{a}, \boldsymbol{b}, \mathrm{GAP}-43^{+/+} ; \boldsymbol{c}-\boldsymbol{e}, \mathrm{GAP}-43^{-/-}$. Arrowheads indicate regions in which Cdc42 and IQGAP1 are colocalized at the base of the primary process. Arrow in c indicates region in which only IQGAP1 is localized. Arrow in $\boldsymbol{d}$ and $\boldsymbol{e}$ indicates region in which Cdc42 and IQGAP1 are colocalized but not at the base of the primary process. Representative images from $n \geq 50$ cells from three independent experiments. Scale bar, $8 \mu \mathrm{m}$. D, Immunoreactivity of $\alpha$-tubulin (green) and Shh (red) in P0 granule cells Shh plus vitronectin protein pattern. $\boldsymbol{a}, \mathrm{GAP}-43^{+/+} ; \boldsymbol{b}, \mathrm{PO} G A P-43^{-/-}$. Arrowhead in $\boldsymbol{a}$ indicates direction of tubulin polymerization associated with the point of contact with the pattern. Arrowhead in $\boldsymbol{b}$ indicates the point of contact with the pattern, and arrow in $\boldsymbol{b}$ indicates that the direction of tubulin polymerization is not associated with the point of contact. Representative images from $n \geq 50$ cells from three independent experiments. Scale bar, $8 \mu \mathrm{m}$. KO, Knock-out; WT, wild type.

GAP-43 and phospholipids demonstrated that this colocalization is attributable to a direct interaction with PIP3. Comparing GAP-43 binding using the pGAP-43-specific antibody (2G12) demonstrated that the GAP-43 bound to PIP3 is primarily the PKC- phosphorylated form. GAP-43 has been shown previously to interact with PIP2 (Laux et al., 2000), but the specific isoforms of PIP2 had not been investigated. The phospholipid binding assay also revealed that pGAP-43 bound selectively to PIP2 $(3,4)$ and $\operatorname{PIP} 2(3,5)$, whereas GAP-43 bound to PIP2 $(4,5)$ was essentially unphosphorylated.

\section{GAP-43 is not required for PIP3 \\ localization in response to extrinsic cues}

To further examine the role of GAP-43 in centrosome positioning toward an extrinsic cue, we performed the micropattern assay in granule cells in which GAP-43 had been genetically deleted $\left(G A P-43^{-/-}\right)$. Figure $8 A$ shows that GAP-43 is required for centrosome positioning by both laminin and vitronectin, whereas Shh was unable to position centrosomes whether or not GAP-43 was present. Comparing PIP3 localization in $G A P-43^{+/+}$and GAP- $43^{-/-}$granule cell precursors in response to vitronectin plus Shh showed that PIP3 was localized toward the pattern whether GAP-43 was present or not (Fig. $8 \mathrm{~B}$ ); hence, PIP3 location is independent of GAP-43 and therefore upstream of GAP-43 function. However, PIP3 is dependent on PKC function: when $\mathrm{PKC}$ is downregulated in response to the PKC inhibitor bisindolylmaleimide, PIP3 fails to be upregulated. The results therefore indicate that, although GAP-43 and PKC activity are required to position the centrosome in response to extrinsic cues, GAP-43 is not required for $\mathrm{PIP} 3$ upregulation in response to extrinsic cues. This suggests that GAP-43 function is downstream of PIP3 and raises the question of whether PKC phosphorylation of GAP-43 occurs in parallel to integrin signaling rather than in a linear pathway.

GAP-43 is required for the colocalization of Cdc42, Par6, and IQGAP1 to the base of the leading process

Together, our results suggest that PKCphosphorylated GAP-43 is an important link between the upstream upregulation of the PI3K pathway in response to laminin and vitronectin and the downstream response that results in centrosome positioning. We therefore investigated how downregulation of GAP-43 affects the localization of Cdc42, Par6, and IQGAP1, all of which have been shown to be important for downstream responses to cell polarization and migration signals (Golub et al., 2004; Golub and Caroni, 2005; Chen et al., 2006a; Yoshimura et al., 2006). Figure 9, $A a$ and $B a$ (arrowheads), shows that, in cells expressing a control EGFP construct, both Cdc42 and Par6 were localized to the 
base of the leading process. In contrast, when GAP-43 was acutely downregulated with an shRNA construct (Mishra et al., 2008), neither Cdc42 nor Par6 localized to the base of the process (Fig. $9 A b, B b$, arrowheads). Similarly, Cdc42 and Par6 also failed to localize appropriately in GAP$43^{-/-}$neurons (supplemental Fig. S4, available at www.jneurosci.org as supplemental material). Hence, failure to express GAP-43 prevents localization of downstream regulators of polarization.

IQGAP1 is important for transducing actin regulatory signals during polarization and in the growth cone. Cdc42 and IQGAP1 were always colocalized at the base of the leading process as well as in the growth cone in GAP-43 $3^{+/+}$granule cell neurons (Fig. 9C). In this case, absence of GAP-43 resulted in two distinct phenotypes: in some GAP-43 $3^{-/-}$cells, Cdc42 and IQGAP1 did not colocalize at either the base of the primary neurite or the growth cone (Fig. $9 C c, \mathrm{Cdc} 42$ arrowhead and IQGAP1 arrow; $C d, \mathrm{Cdc} 42$ arrowhead). In other cells, Cdc42 and IQGAP1 did colocalize but not at the region giving rise to the primary neurite (Fig. $9 \mathrm{Cd}, \mathrm{Ce}$, arrows). The results show that GAP-43 is required for the localization of both Cdc42 and Par6, suggesting that it is upstream of both Cdc42 and Par6 function. They also demonstrate that the correct spatial localization of Cdc42 and IQGAP1 requires GAP-43 to be PKC phosphorylated. Together, they imply that actin regulation is an important aspect of centrosome positioning.

\section{Absence of GAP-43 is associated with abnormal tubulin extension at the centrosome}

We have shown previously that, in the absence of GAP-43, both kinetocore and astral spindles were abnormal in mitosing granule cells, indicating that, in addition to its documented effects on actin polymerization, GAP-43 also affects microtubules (Mishra et al., 2008). We therefore used the micropatterning assay to determine whether the disrupted response to vitronectin or laminin seen in GAP $-43^{-1-}$ granule cells is also accompanied by abnormal distribution of microtubules. Figure $9 D$ shows that, under normal conditions, microtubules are oriented toward the extrinsic cue responsible for positioning the centrosome in the micropatterning assay. When GAP-43 is absent, microtubules are still evident, but they are not oriented toward the region in which the cell contacts the extrinsic cue (Fig. 9D, arrowhead). The results indicate that, in addition to its previously described actin modulatory functions, GAP-43 at the centrosome influences microtubules orientation.

\section{Discussion}

The mechanism(s) by which extrinsic cues are able to determine where the centrosome is positioned and thereby where the axon will extend during neuronal polarization are key to target identification during neuronal development but are not well understood. The goal of the present study was to identify relevant extrinsic cues able to position the centrosome in polarizing granule cell precursors and then to investigate how the underlying signal transduction is coupled to intrinsic regulation of polarity. Previous studies have shown that laminin and vitronectin act in concert with the granule cell mitogen Shh to regulate cell proliferation and differentiation in granule cells (Liesi, 1992; Pons et al., 2001; Blaess et al., 2004), but how they function in the subsequent centrosome positioning that occurs as a prelude to polarization is unclear. We have shown that both laminin and vitronectin can behave as such extrinsic cues and that they use an integrin-mediated transduction mechanism that is dependent both on PI3K and the growth-associated protein GAP-43. When GAP-43 is absent, the cytoskeletal response that results in microtubule organization is disrupted. The results therefore provide an important first step in understanding how extrinsic and intrinsic regulation of neuronal polarity are coupled.

How granule cell proliferation is downregulated for polarization to be initiated is unclear. Our results showed that, at early stages in the differentiation of granule cell neurons in mice (P0-P8), both laminin and vitronectin reduce the proliferative capacity of substrate-associated Shh in vitro and also control positioning of the centrosome to the base of the leading process in slice cultures that most closely mimic in vivo development. Our results also show that positioning of the centrosome to initiate polarization is independent of Shh. They therefore confirm previous studies that both proliferation and differentiation are affected by laminin and vitronectin. Those studies showed that, although laminin deposition in the external granule layer is important for the directional migration of granule cells toward the internal granule layer (Liesi, 1992; Liesi et al., 1995), presenting Shh in solution to cells in conjunction with substrate-associated laminin potentiates its mitogenic effects (Pons 
et al., 2001). In contrast, vitronectin interacts with Shh directly to stimulate granule cell differentiation (Pons and Martí, 2000). We found that, when Shh is presented in a spatially regulated, substrateassociated format, analogous with its association with laminin and vitronectin in the cerebellar ECM in vivo, both laminin and vitronectin inhibit the proliferative effect of Shh, and both laminin and vitronectin are able to position the centrosome at the base of the primary leading process. Our data therefore suggest that, under conditions in which both ECM components and Shh are spatially constrained, granule cell maturation leading to polarization occurs as a two-step process, with cell cycle exit being controlled by laminin and vitronectin via downregulation of Shh-induced mitogenesis and subsequent centrosome positioning being controlled by laminin and vitronectin via upregulation of integrin signaling. The current results with regard to the effect of laminin on granule cell proliferation differ from that of Pons et al. (2001). Although Shh was printed on the substrate in the current study versus in solution as in the case of the Pons study, this is not the only difference between the two studies that needs to be taken into account. One major difference is that the Pons study used P6 rat cerebellar cultures, whereas the current study uses mouse cerebellar cultures. Perhaps related to this, under basal culture conditions, in the absence of Shh, the Pons study saw no BrdU incorporation and therefore no cell proliferation. In contrast, under our culture conditions, we get between 10 and $15 \%$ of the granule cells proliferating without the addition of Shh. This suggests that the response of granule cells may depend on the initial conditions of the cells in vitro and therefore caution should be exercised when comparing results across different culture conditions and across species.

Upregulation of PI3K by integrin receptors (including $\alpha 5 \beta 3$ ) is known to be important in the polarization of hippocampal neurons in vitro (Zheng et al., 2000; Shi et al., 2003; Ménager et al., 2004). However, the mechanisms by which receptormediated activation of PI3K coordinates with downstream cytoskeletal responses is less clear. We too have shown that laminin and vitronectin signaling via cognate integrin receptors causes an upregulation of PI3K-mediated signaling that is required for granule cell polarization. Our finding that the centrosome positioning that underlies polarization is codependent on both upregulation of $\mathrm{PI} 3 \mathrm{~K}$ and $\mathrm{PKC}$-mediated phosphorylation of GAP-43 identifies for the first time a spatially restricted protein that is both directly responsive to integrin signaling and able to modulate the cytoskeleton in response to extrinsic cues. The present study also shows that phosphorylation of GAP-43 results in a change in the binding affinity for distinct phospholipids and suggesting a potential mechanism whereby a spatially restricted extrinsic signal can given rise to a spatially restricted membrane response (Laux et al., 2000; Golub and Caroni, 2005). In particular, we have shown that PIP3, upregulated in response to PI3 kinase activation, appears to be a key player in transduction of the signal from membrane to cytoskeleton. In neutrophils, PIP3mediated activation of Cdc42 and Rac1, which in turn regulates actin dynamics, drives acquisition of cell polarity and subsequent chemotaxis (Wang et al., 2002; Weiner, 2002). Rac-dependent actin polymerization has also been hypothesized to underlie polarization in hippocampal neurons (Shi et al., 2003). We also shown here that PIP3 upregulation in the centrosome area occurs in response to laminin and vitronectin and that inhibition of PI3K prevents centrosome positioning. Moreover, we have demonstrated for the first time that PIP3 binds directly to GAP-43 and selectively to pGAP-43. Given that both PIP3 and GAP-43 are required for centrosome positioning, the results suggest that PIP3 binding to GAP-43 may be required for the downstream response to integrin signaling activated by laminin and vitronectin. The results also show clearly that, in the absence of GAP-43, extrinsic signaling via upregulation of $\mathrm{PI} 3 \mathrm{~K}$ is decoupled from the intrinsic cytoskeletal response. Hence, PIP3 upregulation is independent of GAP-43, although centrosome positioning is GAP-43 dependent. GAP-43 is localized in distinct membrane microdomains involved in generation of signaling complexes. The involvement of such microdomains in the response to laminin and vitronectin deserves investigation.

The question remains as to how the PKC that phosphorylates GAP-43 is activated. PI3K can activate phospholipase C via diacylglycerol lipase, a pathway shown previously to lead to PKC phosphorylation during granule cell differentiation (Meiri et al., 1998). However, GAP-43 is phosphorylated even in the absence of laminin and vitronectin and can also be phosphorylated by $\mathrm{PKC}$ in response to treatment with soluble Shh (Meiri et al., 1998; Shen et al., 2008). Whether Shh plays an indirect role in centrosome positioning via GAP-43 phosphorylation that is not sufficient to induce polarization in the absence of the ECM is unclear.

Previous studies showing that the position of the centrosome can be determined by the orientation of the spindle during the last mitotic division have given rise to a model in which orientation of the future axon is intrinsically driven by microtubule dynamics (Golub et al., 2004; Golub and Caroni, 2005). The model is supported by the finding that several microtubule stabilization factors, such as LIS1 (lissencephaly 1), NDE1 (nudE nuclear distribution gene $\mathrm{E}$ homolog 1), adenomatosis polyposis coli, as well as members of the kinesin superfamily of proteins, also affect cell polarity (Sasaki et al., 2000; Nishimura et al., 2004; Shi et al., 2004; Horiguchi et al., 2006). Other studies demonstrate that Cdc42 and its effector IQGAP1 that are important for polarization anchor microtubules to cortical actin at the membrane (Golub and Caroni, 2005). Cdc42 is particularly key because its activation regulates localization of the Par complex that is essential for the acquisition of cell polarity (Chen et al., 2006a; Yoshimura et al., 2006). Neither of these models, as they stand, account for how the intrinsic regulation of polarization is coupled to the extrinsic cues that are needed to directionally determine polarization as clearly occurs in vivo. Our results here suggest that one clue may lie in the spatial organization of polarizing machinery in lipid microdomains in the membrane that contain not only Cdc42 and IQGAP1 but also GAP-43 (Golub et al., 2004; Golub and Caroni, 2005). The interaction of GAP-43 with Cdc42 and IQGAP1 in lipid microdomains implicates the phospholipid binding properties of GAP-43 as a key element at the intersection between the outside-in signaling via the extrinsic cues that directionally regulates centrosome positioning and the intrinsic cytoskeletal dynamics that underlie centrosome positioning. In this model (Fig. 10), cell polarity in granule cells is acquired through an outside-in signaling pathway that is initiated by extracellular matrix proteins laminin and vitronectin and transduced via integrin-mediated upregulation of PI3K that results in upregulation of PIP3. In parallel with this outside-in signaling, PKC-mediated phosphorylation of GAP-43 enables direct interactions with PIP3 at the membrane. The next step will be to determine whether PKC phosphorylation of GAP-43 is itself extrinsically or intrinsically regulated.

\section{References}

Alberts P, Rudge R, Irinopoulou T, Danglot L, Gauthier-Rouvière C, Galli T (2006) Cdc42 and actin control polarized expression of TI-VAMP vesicles to neuronal growth cones and their fusion with the plasma membrane. Mol Biol Cell 17:1194-1203. 
Blaess S, Graus-Porta D, Belvindrah R, Radakovits R, Pons S, LittlewoodEvans A, Senften M, Guo H, Li Y, Miner JH, Reichardt LF, Müller U (2004) Beta1-integrins are critical for cerebellar granule cell precursor proliferation. J Neurosci 24:3402-3412.

Calderon de Anda F, Gärtner A, Tsai LH, Dotti CG (2008) Pyramidal neuron polarity axis is defined at the bipolar stage. J Cell Sci 121:178-185.

Changelian PS, Meiri K, Soppet D, Valenza H, Loewy A, Willard M (1990) Purification of the growth-associated protein GAP- 43 by reversed phase chromatography: amino acid sequence analysis and cDNA identification. Brain Res 510:259-268.

Chen L, Liao G, Yang L, Campbell K, Nakafuku M, Kuan CY, Zheng Y (2006a) Cdc42 deficiency causes Sonic hedgehog-independent holoprosencephaly. Proc Natl Acad Sci U S A 103:16520-16525.

Chen TJ, Gehler S, Shaw AE, Bamburg JR, Letourneau PC (2006b) Cdc42 participates in the regulation of $\mathrm{ADF} /$ cofilin and retinal growth cone filopodia by brain derived neurotrophic factor. J Neurobiol 66:103-114.

de Anda FC, Pollarolo G, Da Silva JS, Camoletto PG, Feiguin F, Dotti CG (2005) Centrosome localization determines neuronal polarity. Nature 436:704-708

Dent EW, Meiri KF (1992) GAP-43 phosphorylation is dynamically regulated in individual growth cones. J Neurobiol 23:1037-1053.

Dent EW, Meiri KF (1998) Distribution of phosphorylated GAP-43 (neuromodulin) in growth cones directly reflects growth cone behavior. J Neurobiol 35:287-299.

Gao WO, Heintz N, Hatten ME (1991) Cerebellar granule cell neurogenesis is regulated by cell-cell interactions in vitro. Neuron 6:705-715.

Gogolla N, Galimberti I, DePaola V, Caroni P (2006a) Preparation of organotypic hippocampal slice cultures for long-term live imaging. Nat Protoc 1:1165-1171.

Gogolla N, Galimberti I, DePaola V, Caroni P (2006b) Staining protocol for organotypic hippocampal slice cultures. Nat Protoc 1:2452-2456.

Golub T, Caroni P (2005) PI(4,5)P2-dependent microdomain assemblies capture microtubules to promote and control leading edge motility. J Cell Biol 169:151-165.

Golub T, Wacha S, Caroni P (2004) Spatial and temporal control of signaling through lipid rafts. Curr Opin Neurobiol 14:542-550.

Higginbotham H, Tanaka T, Brinkman BC, Gleeson JG (2006) GSK3beta and PKCzeta function in centrosome localization and process stabilization during Slit-mediated neuronal repolarization. Mol Cell Neurosci 32:118-132.

Horiguchi K, Hanada T, Fukui Y, Chishti AH (2006) Transport of PIP3 by GAKIN, a kinesin-3 family protein, regulates neuronal cell polarity. J Cell Biol 174:425-436.

Kenney AM, Cole MD, Rowitch DH (2003) Nmyc upregulation by sonic hedgehog signaling promotes proliferation in developing cerebellar granule neuron precursors. Development 130:15-28.

Laux T, Fukami K, Thelen M, Golub T, Frey D, Caroni P (2000) GAP43, MARCKS, and CAP23 modulate $\mathrm{PI}(4,5) \mathrm{P}(2)$ at plasmalemmal rafts, and regulate cell cortex actin dynamics through a common mechanism. J Cell Biol 149:1455-1472.

Liesi P (1992) Neuronal migration on laminin involves neuronal contact formation followed by nuclear movement inside a preformed process. Exp Neurol 117:103-113.

Liesi P, Hager G, Dodt HU, Seppäl ä I, Zieglgänsberger W (1995) Domainspecific antibodies against the B2 chain of laminin inhibit neuronal migration in the neonatal rat cerebellum. J Neurosci Res 40:199-206.

Luo L, Jan LY, Jan YN (1997) Rho family GTP-binding proteins in growth cone signalling. Curr Opin Neurobiol 7:81-86.

Maier DL, Mani S, Donovan SL, Soppet D, Tessarollo L, McCasland JS, Meiri KF (1999) Disrupted cortical map and absence of cortical barrels in growth-associated protein (GAP)-43 knockout mice. Proc Natl Acad Sci U S A 96:9397-9402.

Martí E, Bovolenta P (2002) Sonic hedgehog in CNS development: one signal, multiple outputs. Trends Neurosci 25:89-96.

Meiri KF, Saffell JL, Walsh FS, Doherty P (1998) Neurite outgrowth stimulated by neural cell adhesion molecules requires growth-associated protein-43 (GAP-43) function and is associated with GAP-43 phosphorylation in growth cones. J Neurosci 18:10429-10437.

Ménager C, Arimura N, Fukata Y, Kaibuchi K (2004) PIP3 is involved in neuronal polarization and axon formation. J Neurochem 89:109-118.

Mishra R, Gupta SK, Meiri KF, Fong M, Thostrup P, Juncker D, Mani S (2008) GAP-43 is key to mitotic spindle control and centrosome-based polarization in neurons. Cell Cycle 7:348-357.

Nishimura T, Kato K, Yamaguchi T, Fukata Y, Ohno S, Kaibuchi K (2004) Role of the PAR-3-KIF3 complex in the establishment of neuronal polarity. Nat Cell Biol 6:328-334.

Pons S, Martí E (2000) Sonic hedgehog synergizes with the extracellular matrix protein vitronectin to induce spinal motor neuron differentiation. Development 127:333-342.

Pons S, Trejo JL, Martínez-Morales JR, Martí E (2001) Vitronectin regulates Sonic hedgehog activity during cerebellum development through CREB phosphorylation. Development 128:1481-1492.

Powell SK, Rivas RJ, Rodriguez-Boulan E, Hatten ME (1997) Development of polarity in cerebellar granule neurons. J Neurobiol 32:223-236.

Sasaki S, Shionoya A, Ishida M, Gambello MJ, Yingling J, Wynshaw-Boris A, Hirotsune S (2000) A LIS1/NUDEL/cytoplasmic dynein heavy chain complex in the developing and adult nervous system. Neuron 28:681696

Sharma SK, Sherff CM, Shobe J, Bagnall MW, Sutton MA, Carew TJ (2003) Differential role of mitogen-activated protein kinase in three distinct phases of memory for sensitization in Aplysia. J Neurosci 23:3899-3907.

Shen Y, Mishra R, Mani S, Meiri KF (2008) Both cell-autonomous and cell non-autonomous functions of GAP-43 are required for normal patterning of the cerebellum in vivo. Cerebellum 7:451-466.

Shi SH, Jan LY, Jan YN (2003) Hippocampal neuronal polarity specified by spatially localized mPar3/mPar6 and PI 3-kinase activity. Cell 112:63-75.

Shi SH, Cheng T, Jan LY, Jan YN (2004) APC and GSK-3beta are involved in mPar3 targeting to the nascent axon and establishment of neuronal polarity. Curr Biol 14:2025-2032.

Solecki DJ, Model L, Gaetz J, Kapoor TM, Hatten ME (2004) Par6alpha signaling controls glial-guided neuronal migration. Nat Neurosci 7:11951203.

Wang F, Herzmark P, Weiner OD, Srinivasan S, Servant G, Bourne HR (2002) Lipid products of PI(3)Ks maintain persistent cell polarity and directed motility in neutrophils. Nat Cell Biol 4:513-518.

Weiner OD (2002) Regulation of cell polarity during eukaryotic chemotaxis: the chemotactic compass. Curr Opin Cell Biol 14:196-202.

Yoshimura T, Arimura N, Kaibuchi K (2006) Signaling networks in neuronal polarization. J Neurosci 26:10626-10630.

Zheng DQ, Woodard AS, Tallini G, Languino LR (2000) Substrate specificity of alpha(v)beta(3) integrin-mediated cell migration and phosphatidylinositol 3-kinase/AKT pathway activation. J Biol Chem 275: 24565-24574.

Zmuda JF, Rivas RJ (1998) The Golgi apparatus and the centrosome are localized to the sites of newly emerging axons in cerebellar granule neurons in vitro. Cell Motil Cytoskeleton 41:18-38.

Zmuda JF, Rivas RJ (2000) Actin filament disruption blocks cerebellar granule neurons at the unipolar stage of differentiation in vitro. J Neurobiol 43:313-328. 